\title{
First-order equivalent to Einstein-Hilbert Lagrangian
}

\author{
M. CASTRILlón LÓPEZ \\ Instituto de Ciencias Matemáticas CSIC-UAM-UC3M-UCM \\ Departamento de Geometría y Topología \\ Facultad de Matemáticas, UCM \\ Avda. Complutense s/n, 28040-Madrid, Spain \\ E-mail: mcastri@mat.ucm.es \\ J. MuÑoz MAsqué \\ Instituto de Física Aplicada, CSIC \\ C/ Serrano 144, 28006-Madrid, Spain \\ E-mail: jaime@iec.csic.es \\ E. Rosado María \\ Departamento de Matemática Aplicada \\ Escuela Técnica Superior de Arquitectura, UPM \\ Avda. Juan de Herrera 4, 28040-Madrid, Spain \\ E-mail: eugenia.rosado@upm.es
}

\begin{abstract}
A first-order Lagrangian $L^{\nabla}$ variationally equivalent to the second-order Einstein-Hilbert Lagrangian is introduced. Such a Lagrangian depends on a symmetric linear connection, but the dependence is covariant under diffeomorphisms. The variational problem defined by $L^{\nabla}$ is proved to be regular and its Hamiltonian formulation is studied, including its covariant Hamiltonian attached to $\nabla$.
\end{abstract}

PACS 2010: 04.20.Cv, 04.20.Fy, 04.50.-h, 11.10.Kk.

Mathematics Subject Classification 2010: 58A20, 58E11, 58E30, 83C05.

Key words: Bundle of metrics, Einstein-Hilbert Lagrangian, jet bundle, linear connection.

Acknowledgements: Supported by Ministerio de Ciencia e Innovación of Spain under grant \#MTM2011-22528. 


\section{Introduction}

Let $p: \mathfrak{M} \rightarrow M$ be the bundle of pseudo-Riemannian metrics of a given signature $\left(n^{+}, n^{-}\right), n^{+}+n^{-}=n=\operatorname{dim} M$, over a connected $C^{\in}$ fty manifold oriented by a volume form $\mathbf{v} \in \Omega^{n}(M)$. The Einstein-Hilbert (or E-H for short) functional is the second-order Lagrangian density $L_{E H} \mathbf{v}$ on $\mathfrak{M}$ defined along a metric $g$ by $s^{g} \mathbf{v}_{g}$, where $s^{g}$ denotes the scalar curvature of $g$ and $\mathbf{v}_{g}$ its Riemannian volume form; namely,

(1)

$$
L_{E H} \circ j^{2} g=\sqrt{\left|\operatorname{det}\left(g_{a b}\right)\right|} g^{j k}\left\{\frac{\partial\left(\Gamma^{g}\right)_{j k}^{i}}{\partial x^{i}}-\frac{\partial\left(\Gamma^{g}\right)_{i k}^{i}}{\partial x^{j}}+\left(\Gamma^{g}\right)_{j k}^{l}\left(\Gamma^{g}\right)_{i l}^{i}-\left(\Gamma^{g}\right)_{i k}^{l}\left(\Gamma^{g}\right)_{j l}^{i}\right\},
$$

where $\left(\Gamma^{g}\right)_{j k}^{i}$ are the Christoffel symbols of the Levi-Civita connection $\nabla^{g}$ of the metric $g$. As is known (e.g., see [1, 3.3.1-3.3.2]), the first-order Lagrangian $L_{1}$ defined along $g$ by $\sqrt{\left|\operatorname{det}\left(g_{a b}\right)\right|} g^{j k}\left(\left(\Gamma^{g}\right)_{i j}^{l}\left(\Gamma^{g}\right)_{k l}^{i}-\left(\Gamma^{g}\right)_{j k}^{l}\left(\Gamma^{g}\right)_{i l}^{i}\right)$ differs from $L_{E H}$ by a divergence term, but unfortunately $L_{1}$ is not an invariantly defined quantity.

Below, a geometrically defined first-order Lagrangian $L^{\nabla}$ (depending on an auxiliary symmetric linear connection $\nabla$ on $M$ ) is introduced, which is variationally equivalent to E-H Lagrangian $L_{E H}$ and, consequently, it has the same Euler-Lagrange equations, namely Einstein's field equations in the vacuum for arbitrary signature. In particular, this explains why the E-H Lagrangian admits a true first-order Hamiltonian formalism.

Although $L^{\nabla}$ depends on an auxiliary symmetric linear connection, this dependence is natural with respect to the action of diffeomorphisms of $M$ on connections and on Lagrangian functions, as proved in section 4 below. This fact justifies the construction of such a Lagrangian and the interest of its existence.

Furthermore, the Lagrangian $L^{\nabla}$ is seen to be regular and its Hamiltonian formulation is studied, computing explicitly its momenta functions and the covariant Hamiltonian attached to $\nabla$ in the sense of [10].

\section{The equivalent Lagrangian $L^{\nabla}$ defined}

The difference tensor field between the Levi-Civita connection $\nabla^{g}$ of a metric $g$ and a given symmetric linear connection $\nabla$ on $M$ is the 2-covariant 1contravariant tensor given by,

$$
T^{g, \nabla}=\nabla^{g}-\nabla=\left(\left(\Gamma^{g}\right)_{i j}^{h}-\Gamma_{i j}^{h}\right) d x^{i} \otimes d x^{j} \otimes \frac{\partial}{\partial x^{h}},
$$

where $\left(\Gamma^{g}\right)_{j k}^{i}$ (resp. $\Gamma_{j k}^{i}$ ) are the Christoffel symbols of the connection $\nabla^{g}$ (resp. $\nabla)$. A Lagrangian function $L^{\nabla}$ on the bundle of metrics $p: \mathfrak{M} \rightarrow M$ is defined as follows:

$$
L^{\nabla}\left(j_{x}^{2} g\right) \mathbf{v}_{x}=\left\{s^{g}(x)+c\left(\left(\operatorname{alt}_{23}\left(\nabla^{g} T^{g, \nabla}\right)_{x}\right)^{\sharp}\right)\right\}\left(\mathbf{v}_{g}\right)_{x}, \quad \forall j_{x}^{2} g \in J^{2} \mathfrak{M},
$$


where we confine ourselves to consider coordinate systems $\left(x^{1}, \ldots, x^{n}\right)$ on $M$ adapted to $\mathbf{v}$, i.e.,

$$
\mathbf{v}=d x^{1} \wedge \ldots \wedge d x^{n}, \quad \mathbf{v}_{g}=\sqrt{\left|\operatorname{det}\left(g_{u v}\right)\right|} \mathbf{v}, \quad g=g_{u v} d x^{u} \otimes d x^{v},
$$

alt $_{23}: \otimes^{3} T^{*} M \otimes T M \rightarrow \otimes^{3} T^{*} M \otimes T M$ denotes the alternation of the second and third covariant indices, ${ }^{\sharp}: \otimes^{3} T^{*} M \otimes T M \rightarrow \otimes^{2} T^{*} M \otimes^{2} T M$ is the isomorphism induced by $g$,

$w_{1} \otimes w_{2} \otimes w_{3} \otimes X \mapsto w_{1} \otimes w_{2} \otimes\left(w_{3}\right)^{\sharp} \otimes X, \quad \forall X \in T_{x} M, \forall w_{1}, w_{2}, w_{3} \in T_{x}^{*} M$,

and, finally, $c: \otimes^{2} T^{*} M \otimes^{2} T M \rightarrow \mathbb{R}$ denotes the (total) contraction of the first and second covariant indices with the first and second contravariant ones, respectively. We write $L^{\nabla}$ in order to emphasize the fact that the Lagrangian depends on the auxiliary symmetric linear connection $\nabla$ previously chosen.

If $y_{i j}=y_{j i}, i, j=1, \ldots, n$, are the coordinates on the fibres of $p$ induced from a coordinate system $\left(x^{h}\right)_{h=1}^{n}$ on $M$, namely, $g_{x}=y_{i j}\left(g_{x}\right) d x^{i} \otimes d x^{j}$ for every metric $g_{x}$ over $x \in M$, and $\left(x^{h}, y_{i j}, y_{i j, k}, y_{i j, k l}=y_{i j, l k}\right)$ denotes the coordinate system induced on $J^{2} \mathfrak{M}$, then $L_{E H}$ is locally given by,

$$
L_{E H}=\rho\left(y^{a c} y^{b d}-y^{a b} y^{c d}\right) y_{a b, c d}+L_{0},
$$

where

$$
\left\{\begin{array}{l}
L_{0}=\rho y^{i j}\left\{y^{h m}\left(y_{m r, j} G_{i h}^{r}-y_{m r, h} G_{i j}^{r}\right)+G_{i j}^{m} G_{h m}^{h}-G_{i h}^{m} G_{j m}^{h}\right\}, \\
\rho=\sqrt{\left|\operatorname{det}\left(y_{i j}\right)\right|},
\end{array}\right.
$$

and $G_{r j}^{i}: J^{1} \mathfrak{M} \rightarrow \mathbb{R}$ are defined by, $G_{r j}^{i}=\frac{1}{2} y^{i s}\left(y_{r s, j}+y_{j s, r}-y_{r j, s}\right)$.

If $L^{\prime \nabla}$ is the second-order Lagrangian on $\mathfrak{M}$ determined by the second summand of the right-hand side in the formula (2), namely

$$
L^{\prime \nabla}\left(j_{x}^{2} g\right)=c\left(\left(\operatorname{alt}_{23}\left(\nabla^{g} T^{g, \nabla}\right)_{x}\right)^{\sharp}\right),
$$

then (2) can equivalently be rewritten as follows: $L^{\nabla}=L_{E H}+\rho L^{\prime \nabla}$ and as a calculation shows,

$$
\begin{aligned}
L^{\prime \nabla} \circ j^{2} g & =g^{j r}\left\{\frac{\partial\left(T^{g, \nabla}\right)_{r i}^{i}}{\partial x^{j}}-\frac{\partial\left(T^{g, \nabla}\right)_{r j}^{i}}{\partial x^{i}}\right. \\
& +\left(\Gamma^{g}\right)_{j i}^{a}\left(T^{g, \nabla}\right)_{r a}^{i}-\left(\Gamma^{g}\right)_{j r}^{a}\left(T^{g, \nabla}\right)_{a i}^{i} \\
& \left.+\left(\Gamma^{g}\right)_{i r}^{a}\left(T^{g, \nabla}\right)_{a j}^{i}-\left(\Gamma^{g}\right)_{a i}^{a}\left(T^{g, \nabla}\right)_{r j}^{i}\right\} .
\end{aligned}
$$

Lemma 2.1. The Lagrangian $L^{\nabla}$ is of first order.

Proof. Taking the definition of $T^{g, \nabla}$ and the formulas (5), (11) into account, one 
obtains

$$
\begin{aligned}
\sqrt{\left|\operatorname{det}\left(g_{u v}\right)\right|}\left(L^{\prime \nabla} \circ j^{2} g\right) & =-L_{E H} \circ j^{2} g \\
& +\sqrt{\left|\operatorname{det}\left(g_{u v}\right)\right|} g^{j r}\left\{\left(\Gamma^{g}\right)_{j i}^{a}\left(\Gamma^{g}\right)_{r a}^{i}-\left(\Gamma^{g}\right)_{a i}^{a}\left(\Gamma^{g}\right)_{r j}^{i}\right\} \\
& -\sqrt{\left|\operatorname{det}\left(g_{u v}\right)\right|} g^{j r}\left\{\frac{\partial \Gamma_{r i}^{i}}{\partial x^{j}}-\frac{\partial \Gamma_{r j}^{i}}{\partial x^{i}}+\left(\Gamma^{g}\right)_{j i}^{a} \Gamma_{r a}^{i}\right. \\
& \left.-\left(\Gamma^{g}\right)_{j r}^{a} \Gamma_{a i}^{i}+\left(\Gamma^{g}\right)_{i r}^{a} \Gamma_{a j}^{i}-\left(\Gamma^{g}\right)_{a i}^{a} \Gamma_{r j}^{i}\right\} .
\end{aligned}
$$

Hence $\left(\rho L^{\prime \nabla}+L_{E H}\right) \circ j^{2} g$ depends on the values of the metric $g$ and its first derivatives only.

In fact, the following local expression is readily deduced:

$$
L^{\nabla}=\rho y^{j r}\left\{G_{j i}^{a} T_{r a}^{i}-G_{a i}^{a} T_{r j}^{i}+G_{j r}^{a} \Gamma_{a i}^{i}-G_{i r}^{a} \Gamma_{a j}^{i}-\frac{\partial \Gamma_{r i}^{i}}{\partial x^{j}}+\frac{\partial \Gamma_{r j}^{i}}{\partial x^{i}}\right\}
$$

$T_{j k}^{i}: J^{1} \mathfrak{M} \rightarrow \mathbb{R}$ being the functions defined by, $T_{i j}^{h}=G_{i j}^{h}-\Gamma_{i j}^{h}$.

Remark 2.1. As $L^{\nabla}$ has a geometrical definition, the local expression above actually provides a global Lagrangian. Moreover, if $\nabla$ is a flat linear connection and one considers an adapted coordinate system to $\nabla$ (i.e., a coordinate system on which all the Christoffel symbols of $\nabla$ vanish), then the local expression for $L^{\nabla}$ coincides with the local Lagrangian $L_{1}$ defined in the introductory section.

\section{$3 \quad L^{\nabla}$ and $L_{E H}$ are variationally equivalent}

As a computation shows, the second summand in the definition of $L^{\nabla}$ can be rewritten in terms of the metric $g$ and the auxiliary connection $\nabla$ only, as follows:

$$
\begin{aligned}
c\left(\left(\operatorname{alt}_{23}\left(\operatorname{nabla}^{g} T^{g, \nabla}\right)\right)^{\sharp}\right) & =\left(g^{j s} g^{i r}-g^{j r} g^{i s}\right) g_{r i, s j} \\
& +\frac{1}{2}\left\{\left(2 g^{i r} g^{j b}-g^{b i} g^{r j}-g^{b r} g^{i j}\right) g^{a s}\right. \\
& +\left(g^{a r} g^{i b}+g^{b i} g^{r a}-2 g^{i r} g^{a b}\right) g^{j s} \\
& -\left(g^{s r} g^{j b}-g^{b r} g^{s j}\right) g^{a i} \\
& \left.-\left(g^{a r} g^{s b}-g^{s r} g^{a b}\right) g^{i j}\right\} g_{a b, j} g_{r s, i} \\
& -g^{j r}\left(\frac{\partial \Gamma_{r i}^{i}}{\partial x^{j}}-\frac{\partial \Gamma_{r j}^{i}}{\partial x^{i}}\right) \\
& +\frac{1}{2}\left\{\left(2 g^{j s} g^{a r}-g^{j r} g^{a s}\right) g_{r j, s} \Gamma_{a i}^{i}\right. \\
& \left.+\left(g^{j r} g^{a b}-2 g^{a r} g^{j b}\right) g_{a b, i} \Gamma_{r j}^{i}\right\} .
\end{aligned}
$$

Lemma 3.1. If $D_{i}$ denotes the total derivative with respect to $x^{i}$, then

$$
c\left(\left(\operatorname{alt}_{23}\left(\nabla^{g} T^{g, \nabla}\right)\right)^{\sharp}\right) \mathbf{v}_{g}=-\left(D_{i}\left(\left(L_{E H}\right)_{\nabla}^{i}\right) \circ j^{2} g\right) \mathbf{v},
$$


where

$$
\left(L_{E H}\right)_{\nabla}^{i}=\sum_{c \leq r} \frac{1}{2-\delta_{i b}} \frac{\partial L_{E H}}{\partial y_{c r, i b}}\left(y_{c r, b}-\left(\Gamma_{b c}^{a} y_{a r}+\Gamma_{b r}^{a} y_{a c}\right)\right) .
$$

From this lemma it follows that $L^{\nabla}$ and $L_{E H}$ are variationally equivalent as, according to the formula (2), one has

$$
\begin{aligned}
\left(L^{\nabla} \circ j^{2} g\right) \mathbf{v} & =\left(L_{E H} \circ j^{2} g\right) \mathbf{v}+c\left(\left(\operatorname{alt}_{23}\left(\nabla^{g} T^{g, \nabla}\right)\right)^{\sharp}\right) \mathbf{v}_{g} \\
& =\left\{\left(L_{E H}-D_{i}\left(\left(L_{E H}\right)_{\nabla}^{i}\right)\right) \circ j^{2} g\right\} \mathbf{v} .
\end{aligned}
$$

Hence $L^{\nabla}=L_{E H}-D_{i}\left(\left(L_{E H}\right)_{\nabla}^{i}\right)$ and therefore, $L^{\nabla}$ and $L_{E H}$ differ in a total divergence.

The proof of Lemma 3.1 follows by computing $D_{i}\left(\left(L_{E H}\right)_{\nabla}^{i}\right)$ using (3) and (7), taking the identity $D_{i} \rho=\frac{\rho}{2} y^{r s} y_{r s, i}$ into account, after a simple-but rather long - computation.

\section{Dependence on $\nabla$}

Below, the dependence of the Lagrangian $L^{\nabla}$ with respect to the symmetric linear connection $\nabla$, is analysed. First, some geometric preliminaries are introduced.

The image of a linear connection $\nabla$ by a diffeomorphism $\phi: M \rightarrow M$ is defined to be $(\phi \cdot \nabla)_{X} Y=\phi \cdot\left(\nabla_{\phi^{-1} \cdot X}\left(\phi^{-1} \cdot Y\right)\right), \forall X, Y \in \mathfrak{X}(M)$. As is well known (e.g., see [4, p. 643]), the Levi-Civita connection of a metric transforms according to the rule: $\phi^{-1} \cdot \nabla^{g}=\nabla^{\phi^{*}} g$. Hence the following formulas hold:

$$
\phi^{-1} \cdot T^{g, \nabla}=T^{\phi^{*} g, \phi^{-1} \cdot \nabla}, \quad S^{\phi \cdot \nabla}=\left(\phi^{-1}\right)^{*} S^{\nabla}=\phi \cdot S^{\nabla}, \quad s^{g}=s^{\phi^{*} g},
$$

where $S^{\nabla}(X, Y)=\operatorname{trace}\left(Z \mapsto R^{\nabla}(Z, X) Y\right)$ is the Ricci tensor of $\nabla$ (e.g., see [7, VI, p. 248]). Moreover, the lift of $\phi$ to the bundle of metrics $p: \mathfrak{M} \rightarrow M$ is given by $\bar{\phi}\left(g_{x}\right)=\left(\phi^{-1}\right)^{*} g_{x}, \forall g_{x} \in p^{-1}(x)$ (cf. [11]); hence $p \circ \bar{\phi}=\phi \circ p$, and the mapping $\bar{\phi}: \mathfrak{M} \rightarrow \mathfrak{M}$ has an extension to the $r$-jet bundle $\bar{\phi}^{(r)}: J^{r} \mathfrak{M} \rightarrow J^{r} \mathfrak{M}$ defined by, $\bar{\phi}^{(r)}\left(j_{x}^{r} g\right)=j_{\phi(x)}^{r}\left(\bar{\phi} \circ g \circ \phi^{-1}\right)$.

Let $\mathbf{v}_{\mathfrak{M}}$ be the nowhere-vanishing $p$-horizontal $n$-form on $\mathfrak{M}$ defined as follows: $\left(\mathbf{v}_{\mathfrak{M}}\right)_{g_{x}}=\mathbf{v}_{g_{x}}, \forall g_{x} \in \mathfrak{M}$, where, as above, $\mathbf{v}_{g_{x}}$ denotes the Riemannian volume form attached to $g_{x}$. Hence $\mathbf{v}_{\mathfrak{M}}=\rho \mathbf{v}$, where $\rho$ is as in (4). Every $r$-th order Lagrangian density $\Lambda$ on $\mathfrak{M}$ can thus be written as $\Lambda=L \mathbf{v}_{\mathfrak{M}}$ for a certain Lagrangian function $L \in C^{\infty}\left(J^{r} \mathfrak{M}\right)$ and $\Lambda$ is invariant under diffeomorphisms, i.e., $\left(\bar{\phi}^{(r)}\right)^{*} \Lambda=\Lambda, \forall \phi \in$ Diff $M$, if and only if $L$ is, i.e., $L \circ \bar{\phi}^{(r)}=L$, as $\left(\bar{\phi}^{(r)}\right)^{*} \Lambda=\left(L \circ \bar{\phi}^{(r)}\right)\left(\bar{\phi}^{*} \mathbf{v}_{\mathfrak{M}}\right)$ and, according to [12, Proposition 7], $\mathbf{v}_{\mathfrak{M}}$ is invariant under diffeomorphisms, i.e., $\bar{\phi}^{*} \mathbf{v}_{\mathfrak{M}}=\mathbf{v}_{\mathfrak{M}}$.

The E-H Lagrangian density $L_{E H} \mathbf{v}$ is known to be invariant under diffeomorhisms, i.e., $\left(\bar{\phi}^{(2)}\right)^{*}\left(L_{E H} \mathbf{v}\right)=L_{E H} \mathbf{v}, \forall \phi \in$ Diff $M$. In fact, there exists a 
classical result by Hermann Weyl ([14, Appendix II], also see [5], [8]), according to which the only Diff $M$-invariant Lagrangians on $J^{2} \mathfrak{M}$ depending linearly on the second-order coordinates $y_{a b, i j}$ are of the form $\lambda L_{E H}+\mu$, for scalars $\lambda, \mu$.

Therefore, transforming the equation $L^{\nabla} \mathbf{v}=L_{E H} \mathbf{v}+L^{\prime \nabla_{\mathfrak{M}}}$ by a diffeomorphism $\phi$, one obtains $\left(\bar{\phi}^{(1)}\right)^{*}\left(L^{\nabla} \mathbf{v}\right)=L_{E H} \mathbf{v}+\left(L^{\prime \nabla} \circ \bar{\phi}^{(2)}\right) \mathbf{v}_{\mathfrak{M}}$, and one is led to compute $L^{\prime \nabla} \circ \bar{\phi}^{(2)}$, which, by using the formulas above, is proved to transform according to the following rule:

$$
L^{\prime \nabla} \circ \bar{\phi}^{(2)}=L^{\prime \phi^{-1} \cdot \nabla}
$$

\section{Hamiltonian formalism}

\section{$5.1 \quad$ Regularity of $L^{\nabla}$}

Proposition 5.1. For $\operatorname{dim} M=n \geq 3$, the Lagrangian $L^{\nabla}$ is regular, namely, the following square matrix of size $\frac{1}{2} n^{2}(n+1)$ is non-singular:

$$
\left(\frac{\partial p_{w}^{u v}}{\partial y_{a b, c}}\right)_{a \leq b, c}^{u \leq v, w}=\left(\frac{\partial^{2} H^{\nabla}}{\partial y_{a b, c} \partial y_{u v, w}}\right)_{a \leq b, c}^{u \leq v, w}
$$

where

$$
p_{k}^{i j}=\frac{\partial L^{\nabla}}{\partial y_{i j, k}}, \quad H^{\nabla}=\sum_{i \leq j} \frac{\partial L^{\nabla}}{\partial y_{i j, k}} y_{i j, k}-L^{\nabla} .
$$

Proof. From the very definition of $H^{\nabla}$ it follows:

$$
\frac{\partial H^{\nabla}}{\partial y_{a b, c}}=\sum_{i \leq j} \frac{\partial^{2} L^{\nabla}}{\partial y_{a b, c} \partial y_{i j, k}} y_{i j, k}
$$

and the formula (9) above. Moreover, we claim that the functions $p_{w}^{u v}$ depend linearly on the variables $y_{a b, c}$. In fact, as a calculation shows,

$$
\begin{aligned}
\frac{\partial p_{w}^{u v}}{\partial y_{a b, c}} & =\frac{\partial^{2} L^{\nabla}}{\partial y_{a b, c} \partial y_{u v, w}} \\
& =\rho y^{j r} \frac{\partial^{2}}{\partial y_{a b, c} \partial y_{u v, w}}\left(G_{j i}^{l} G_{r l}^{i}-G_{l i}^{l} G_{r j}^{i}\right) \\
& =\frac{1}{\left(1+\delta_{a b}\right)\left(1+\delta_{u v}\right)} \rho\left\{y^{b w}\left(y^{a u} y^{c v}+y^{a v} y^{c u}\right)+y^{a w}\left(y^{b u} y^{c v}+y^{b v} y^{c u}\right)\right. \\
& -y^{a b}\left(y^{c u} y^{v w}+y^{c v} y^{u w}\right)-y^{u v}\left(y^{a w} y^{b c}+y^{a c} y^{b w}\right) \\
& \left.-\left(y^{u a} y^{v b}+y^{u b} y^{v a}\right) y^{w c}+2 y^{a b} y^{u v} y^{w c}\right\} .
\end{aligned}
$$

Therefore, in order to prove that the matrix (9) is non-singular, it suffices to prove that the variables $y_{a b, c}$ can be written in terms of the functions $p_{w}^{u v}$. To 
do this, we first compute

$$
\begin{aligned}
\sum_{u, v, w} \frac{1+\delta_{u v}}{\rho} p_{w}^{u v} y_{u r} y_{v s} y_{w q} & =y_{q r, s}+y_{q s, r}-y_{r s, q} \\
& -\frac{1}{2} \sum_{a, b} y^{a b}\left(y_{s q} y_{a b, r}+y_{r q} y_{a b, s}\right) \\
& +\sum_{a, b} y^{a b} y_{r s}\left(y_{a b, q}-y_{q a, b}\right) .
\end{aligned}
$$

Evaluating the previous formula at $g_{x_{0}}$, by using adapted coordinates (i.e., $y_{i j}\left(g_{x_{0}}\right)=\varepsilon_{i} \delta_{i j}, \varepsilon_{i}= \pm 1$ ), and letting $\Upsilon_{r s q}\left(j_{x_{0}}^{1} g\right)=\frac{1+\delta_{r s}}{\rho} p_{q}^{r s}\left(j_{x_{0}}^{1} g\right) \varepsilon_{r} \varepsilon_{s} \varepsilon_{q}$, it follows:

$$
\begin{aligned}
\Upsilon_{r s q}\left(j_{x_{0}}^{1} g\right) & =y_{q r, s}\left(j_{x_{0}}^{1} g\right)+y_{q s, r}\left(j_{x_{0}}^{1} g\right)-y_{r s, q}\left(j_{x_{0}}^{1} g\right) \\
& -\frac{1}{2} \sum_{a} \varepsilon_{a} \varepsilon_{q}\left(\delta_{s q} y_{a a, r}\left(j_{x_{0}}^{1} g\right)+\delta_{r q} y_{a a, s}\left(j_{x_{0}}^{1} g\right)\right) \\
& +\sum_{a} \varepsilon_{a} \varepsilon_{r} \delta_{r s}\left(y_{a a, q}\left(j_{x_{0}}^{1} g\right)-y_{q a, a}\left(j_{x_{0}}^{1} g\right)\right) .
\end{aligned}
$$

If $q \neq r \neq s \neq q$, then $\Upsilon_{r s q}\left(j_{x_{0}}^{1} g\right)=y_{q r, s}\left(j_{x_{0}}^{1} g\right)+y_{q s, r}\left(j_{x_{0}}^{1} g\right)-y_{r s, q}\left(j_{x_{0}}^{1} g\right)$. Hence

$$
y_{q r, s}\left(j_{x_{0}}^{1} g\right)=\frac{1}{2}\left(\Upsilon_{r s q}\left(j_{x_{0}}^{1} g\right)+\Upsilon_{q s r}\left(j_{x_{0}}^{1} g\right)\right) .
$$

If $q=r, r \neq s$, then

$$
\Upsilon_{r s r}\left(j_{x_{0}}^{1} g\right)=y_{r r, s}\left(j_{x_{0}}^{1} g\right)-\frac{1}{2} \sum_{a} \varepsilon_{a} \varepsilon_{r} y_{a a, s}\left(j_{x_{0}}^{1} g\right) .
$$

If $r=s, q \neq r$, then

$$
\begin{aligned}
\Upsilon_{r r q}\left(j_{x_{0}}^{1} g\right) & =2 y_{q r, r}\left(j_{x_{0}}^{1} g\right)-y_{r r, q}\left(j_{x_{0}}^{1} g\right) \\
& +\sum_{a} \varepsilon_{a} \varepsilon_{r}\left(y_{a a, q}\left(j_{x_{0}}^{1} g\right)-y_{q a, a}\left(j_{x_{0}}^{1} g\right)\right) .
\end{aligned}
$$

The formula (12) can be rewritten as

$$
2 \varepsilon_{r} \Upsilon_{r s r}\left(j_{x_{0}}^{1} g\right)=\varepsilon_{r} y_{r r, s}\left(j_{x_{0}}^{1} g\right)-\sum_{a \neq r} \varepsilon_{a} y_{a a, s}\left(j_{x_{0}}^{1} g\right) .
$$

Summing up over the index $r, 2 \sum_{r} \varepsilon_{r} \Upsilon_{r s r}\left(j_{x_{0}}^{1} g\right)=(2-n) \sum_{r} \varepsilon_{r} y_{r r, s}\left(j_{x_{0}}^{1} g\right)$, and replacing this formula into (12) it follows:

$$
\Upsilon_{r s r}\left(j_{x_{0}}^{1} g\right)=y_{r r, s}\left(j_{x_{0}}^{1} g\right)-\frac{1}{2-n} \varepsilon_{r} \sum_{a} \varepsilon_{a} \Upsilon_{a s a}\left(j_{x_{0}}^{1} g\right)
$$

Therefore

$$
y_{r r, s}\left(j_{x_{0}}^{1} g\right)=\Upsilon_{r s r}\left(j_{x_{0}}^{1} g\right)+\frac{\varepsilon_{r}}{2-n} \sum_{a} \varepsilon_{a} \Upsilon_{a s a}\left(j_{x_{0}}^{1} g\right) .
$$

Replacing (14) into (13), we eventually obtain

$$
\sum_{a} \varepsilon_{a} y_{q a, a}\left(j_{x_{0}}^{1} g\right)=\frac{1}{n-2} \sum_{a} \varepsilon_{a} \Upsilon_{a a q}\left(j_{x_{0}}^{1} g\right)-2 \frac{n-1}{(n-2)^{2}} \sum_{a} \varepsilon_{a} \Upsilon_{a q a}\left(j_{x_{0}}^{1} g\right)
$$


and replacing $y_{r r, q}\left(j_{x_{0}}^{1} g\right), \sum_{a} \varepsilon_{a} y_{a a, q}\left(j_{x_{0}}^{1} g\right)$, and $\sum_{a} \varepsilon_{a} y_{q a, a}\left(j_{x_{0}}^{1} g\right)$ into (13) it follows:

$$
\begin{aligned}
\Upsilon_{r r q}\left(j_{x_{0}}^{1} g\right) & =2 y_{q r, r}\left(j_{x_{0}}^{1} g\right)-\Upsilon_{r q r}\left(j_{x_{0}}^{1} g\right)+\frac{n \varepsilon_{r}}{(n-2)^{2}} \sum_{a} \varepsilon_{a} \Upsilon_{a q a}\left(j_{x_{0}}^{1} g\right) \\
& -\frac{\varepsilon_{r}}{n-2} \sum_{a} \varepsilon_{a} \Upsilon_{a a q}\left(j_{x_{0}}^{1} g\right) .
\end{aligned}
$$

Hence

$$
\begin{aligned}
y_{q r, r}\left(j_{x_{0}}^{1} g\right) & =\frac{1}{2} \frac{n-1}{n-2} \Upsilon_{r r q}\left(j_{x_{0}}^{1} g\right)+\frac{1}{2}\left(1-\frac{n}{(n-2)^{2}}\right) \Upsilon_{r q r}\left(j_{x_{0}}^{1} g\right) \\
& -\frac{n \varepsilon_{r}}{2(n-2)^{2}} \sum_{a \neq r} \varepsilon_{a} \Upsilon_{a q a}\left(j_{x_{0}}^{1} g\right) \\
& +\frac{\varepsilon_{r}}{2(n-2)} \sum_{a \neq r} \varepsilon_{a} \Upsilon_{a a q}\left(j_{x_{0}}^{1} g\right)
\end{aligned}
$$

If $q=r=s$, then $\Upsilon_{r r r}\left(j_{x_{0}}^{1} g\right)=-\sum_{a \neq r} \varepsilon_{a} \varepsilon_{r} y_{r a, a}\left(j_{x_{0}}^{1} g\right)$. From (15) we obtain $\sum_{a \neq r} \varepsilon_{a} y_{r a, a}\left(j_{x_{0}}^{1} g\right)$ and then

$$
\begin{aligned}
\sum_{a \neq r} \varepsilon_{a} y_{r a, a}\left(j_{x_{0}}^{1} g\right) & =-\varepsilon_{r} y_{r r, r}\left(j_{x_{0}}^{1} g\right)+\frac{1}{n-2} \sum_{a} \varepsilon_{a} \Upsilon_{a a r}\left(j_{x_{0}}^{1} g\right) \\
& -2 \frac{n-1}{(n-2)^{2}} \sum_{a} \varepsilon_{a} \Upsilon_{a r a}\left(j_{x_{0}}^{1} g\right)
\end{aligned}
$$

and replacing it into the previous equation,

$$
\Upsilon_{r r r}\left(j_{x_{0}}^{1} g\right)=y_{r r, r}\left(j_{x_{0}}^{1} g\right)-\frac{\varepsilon_{r}}{n-2} \sum_{a} \varepsilon_{a} \Upsilon_{a a r}\left(j_{x_{0}}^{1} g\right)+2 \frac{(n-1) \varepsilon_{r}}{(n-2)^{2}} \sum_{a} \varepsilon_{a} \Upsilon_{a r a}\left(j_{x_{0}}^{1} g\right)
$$

Hence

$$
\begin{aligned}
y_{r r, r}\left(j_{x_{0}}^{1} g\right) & =\Upsilon_{r r r}\left(j_{x_{0}}^{1} g\right)+\varepsilon_{r} \frac{1}{n-2} \sum_{a} \varepsilon_{a} \Upsilon_{a a r}\left(j_{x_{0}}^{1} g\right) \\
& -2 \varepsilon_{r} \frac{n-1}{(n-2)^{2}} \sum_{a} \varepsilon_{a} \Upsilon_{a r a}\left(j_{x_{0}}^{1} g\right) .
\end{aligned}
$$

The formulas (11), (14), (16), and (17) end the proof.

\subsection{Hamilton-Cartan equations}

The Poincaré-Cartan form for the density $L^{\nabla} \mathbf{v}$ is the $n$-form on $J^{1} \mathfrak{M}$ given by

$$
\Theta_{L \nabla \mathbf{v}}=\sum_{i \leq j}(-1)^{k-1} p_{k}^{i j} d y_{i j} \wedge \mathbf{v}_{k}-H^{\nabla} \mathbf{v}
$$

the momenta $p_{k}^{i j}$ and the Hamiltonian function $H^{\nabla}$ being defined as in (10), and the Hamilton-Cartan equations can geometrically be written as

$$
\left(j^{1} g\right)^{*}\left(i_{Y} d \Theta_{L \nabla \mathbf{v}}\right)=0,
$$

for every $p^{1}$-vertical vector field $Y \in J^{1} \mathfrak{M}$, which are known to be equivalent to Euler-Lagrange equations, where $p^{1}: J^{1} \mathfrak{M} \rightarrow M$ is the natural projection. 
According to Proposition [5.1 $\left(x^{i}, y_{j k}, p_{w}^{u v}\right), j \leq k, u \leq v$, is a coordinate system on $J^{1} \mathfrak{M}$. Letting $Y=\partial / \partial y_{a b}$ and $Y=\partial / \partial p_{w}^{u v}$ in (18), it follows respectively:

$$
\begin{gathered}
\sum_{k} \frac{\partial\left(p_{k}^{a b} \circ j^{1} g\right)}{\partial x^{k}}=-\frac{\partial H^{\nabla}}{\partial y_{a b}} \circ j^{1} g, \\
\frac{\partial\left(y_{u v} \circ j^{1} g\right)}{\partial x^{w}}=\frac{\partial H^{\nabla}}{\partial p_{w}^{u v}} \circ j^{1} g,
\end{gathered}
$$

which are the Hamilton-Cartan equations in the canonical formalism.

\subsection{Covariant Hamiltonian}

An Ehresmann (or non-linear) connection on a fibred manifold $p: E \rightarrow M$ is a differential 1-form $\gamma$ on $E$ taking values in the vertical sub-bundle $V(p)$ such that $\gamma(X)=X$ for every $X \in V(p)$, e.g., see [9, 10, 13]. Given $\gamma$, one has $T(E)=V(p) \oplus \operatorname{ker} \gamma, \operatorname{ker} \gamma$ being the horizontal sub-bundle attached to $\gamma$.

According to [10, the covariant Hamiltonian $\mathcal{H}^{\gamma}$ associated to a Lagrangian density $\Lambda$ on $J^{1} E$ with respect to $\gamma$ is the Lagrangian density defined by setting $\mathcal{H}^{\gamma}=\left(\left(p_{0}^{1}\right)^{*} \gamma-\theta\right) \wedge \omega_{\Lambda}-\Lambda$, where $p^{1}: J^{1} E \rightarrow M, p_{0}^{1}: J^{1} E \rightarrow J^{0} E=E$ are the natural projections, and $\omega_{\Lambda}$ is the Legendre form attached to $\Lambda$, i.e., the $V^{*}(p)$-valued $p^{1}$-horizontal $(n-1)$-form on $J^{1} E$ given by

$$
\omega_{\Lambda}=(-1)^{i-1} \frac{\partial L}{\partial y_{i}^{\alpha}} d x^{1} \wedge \cdots \wedge \widehat{d x^{i}} \wedge \cdots \wedge d x^{n} \otimes d y^{\alpha}, \quad \Lambda=L \mathbf{v},
$$

and $\theta=\theta^{\alpha} \otimes \partial / \partial y^{\alpha}, \theta^{\alpha}=d y^{\alpha}-y_{i}^{\alpha} d x^{i}$, is the $V(p)$-valued contact 1-form on $J^{1} E$. Locally, $\mathcal{H}^{\gamma}=\left(\left(\gamma_{i}^{\alpha}+y_{i}^{\alpha}\right) \frac{\partial L}{\partial y_{i}^{\alpha}}-L\right) \mathbf{v}$.

Let $\pi: F(M) \rightarrow M$ be the bundle of linear frames and let $q: F(M) \rightarrow \mathfrak{M}$ be the projection given by $q\left(X_{1}, \ldots, X_{n}\right)=g_{x}=\varepsilon_{h} w^{h} \otimes w^{h}$, where $\left(w^{1}, \ldots, w^{n}\right)$ is the dual coframe of $\left(X_{1}, \ldots, X_{n}\right) \in F_{x}(M)$, i.e., $g_{x}$ is the metric for which $\left(X_{1}, \ldots, X_{n}\right)$ is a $g_{x^{-}}$orthonormal basis and $\varepsilon_{h}=1$ for $1 \leq h \leq n^{+}, \varepsilon_{h}=-1$ for $n^{+}+1 \leq h \leq n$. The projection $q$ is a principal $G$-bundle with $G=O\left(n^{+}, n^{-}\right)$. Given a symmetric linear connection $\Gamma$ with associated covariant derivative $\nabla$, and a tangent vector $X \in T_{x} M$, for every $u \in \pi^{-1}(x)$ there exists a unique $\Gamma$-horizontal tangent vector $X_{u}^{h_{\Gamma}} \in T_{u}(F M)$ such that, $\pi_{*} X_{u}^{h_{\Gamma}}=X$. Given a metric $g_{x} \in q^{-1}(x)$, let $u \in \pi^{-1}(x)$ be a linear frame such that $q(u)=g_{x}$. The projection $q_{*}\left(X_{u}^{h_{\Gamma x}}\right)$ does not depend on the linear frame $u$ chosen over $g_{x}$; we refer the reader to [11, Lemma 3.3] for a proof of this fact. In this way a section $\sigma^{\nabla}: p^{*} T M \rightarrow T \mathfrak{M}$ of the projection $p_{*}: T \mathfrak{M} \rightarrow p^{*} T M$ is defined by setting $\sigma^{\nabla}\left(g_{x}, X\right)=q_{*}\left(X_{u}^{h_{\Gamma x}}\right)$. The retract $\gamma^{\nabla}: T \mathfrak{M} \rightarrow V(p)$ associated to $\sigma^{\nabla}$, namely, $\gamma^{\nabla}(Y)=Y-\sigma^{\nabla}\left(p_{*} Y\right), \forall Y \in T_{g_{x}} \mathfrak{M}$, determines an Ehresmann connection on the bundle of metrics and the Lagrangian density $\Lambda^{\nabla}=L^{\nabla} \mathbf{v}$ admits a "canonical" covariant Hamiltonian $\mathcal{H}^{\gamma^{\nabla}}$. Locally,

$$
\gamma^{\nabla}\left(g_{x}, \partial / \partial x^{j}\right)=-\sum_{k \leq l}\left\{\Gamma_{j k}^{a}(x) y_{a l}\left(g_{x}\right)+\Gamma_{j l}^{a}(x) y_{a k}\left(g_{x}\right)\right\}\left(\partial / \partial y_{k l}\right)_{g_{x}} .
$$


Hence, $\gamma_{k l, j}=-\left(\Gamma_{j k}^{a} y_{a l}+\Gamma_{j l}^{a} y_{a k}\right)$, and

$$
\mathcal{H}^{\gamma^{\nabla}}=\left(\sum_{k \leq l}\left(y_{k l, j}-\left(\Gamma_{j k}^{a} y_{a l}+\Gamma_{j l}^{a} y_{a k}\right)\right) \frac{\partial L^{\nabla}}{\partial y_{k l, j}}-L^{\nabla}\right) \mathbf{v} .
$$

From a direct computation the following result is deduced:

$$
\text { If } \mathcal{H}^{\gamma^{\nabla}}=H^{\gamma^{\nabla}} \mathbf{v} \text {, then }
$$

$$
H^{\gamma^{\nabla}}\left(j_{x}^{1} g\right)=L^{\nabla}\left(j_{x}^{1} g\right)-2 \rho\left(g_{x}\right) s^{g, \nabla}(x), \quad \forall j_{x}^{1} g \in J_{x}^{1} \mathfrak{M},
$$

where $s^{g, \nabla}$ is the scalar curvature of the symmetric linear connection $\nabla$ with respect to the metric $g$, namely

$$
s^{g, \nabla}=g^{j k}\left\{\frac{\partial \Gamma_{j k}^{i}}{\partial x^{i}}-\frac{\partial \Gamma_{i k}^{i}}{\partial x^{j}}+\Gamma_{j k}^{l} \Gamma_{i l}^{i}-\Gamma_{i k}^{l} \Gamma_{j l}^{i}\right\} .
$$

The Hamilton-Cartan equations for a covariant Hamiltonian $H^{\gamma}$ attached to a connection $\gamma$ are

$$
\begin{aligned}
\sum_{k} \frac{\partial\left(p_{k}^{a b} \circ j^{1} g\right)}{\partial x^{k}}-\sum_{u \leq v}\left(\frac{\partial \gamma_{u v, w}}{\partial y_{a b}} \circ g\right)\left(p_{w}^{u v} \circ j^{1} g\right) & =-\frac{\partial H^{\gamma}}{\partial y_{a b}} \circ j^{1} g \\
\frac{\partial\left(y_{u v} \circ j^{1} g\right)}{\partial x^{w}}+\gamma_{u v, w} \circ j^{1} g & =\frac{\partial H^{\gamma}}{\partial p_{w}^{u v}} \circ j^{1} g
\end{aligned}
$$

(for example see 2]). Note that for $\gamma=0$ (that is, the trivial connection induced by the coordinate system) these equations coincide with the local expression of the Hamilton-Cartan equations for $H^{\nabla}$ given in $\S 5.2$.

\section{Conclusions}

We have defined a first-order Lagrangian $L^{\nabla}$ on the bundle of metrics which is variationally equivalent to the second-order classical Einstein-Hilbert Lagrangian.

This Lagrangian depends on an auxiliary symmetric linear connection, but this dependence is covariant under the action of the group of diffeomorphisms.

We have also proved that the variational problem defined by $L^{\nabla}$ is regular and its Hamiltonian formulation has been studied, including the covariant Hamiltonian attached to $\nabla$.

Moreover, we shoud finally mention the completely different behaviour of $L^{\nabla}$ with respect to the Palatini Lagrangian.

Let $q: \mathfrak{C} \rightarrow M$ be the bundle of symmetric linear connections on $M$. The Palatini variational principle consists in coupling a metric $g$ and a symmetric linear connection $\nabla$ as independent fields, thus defining a first-order Lagrangian density $L_{P} \mathbf{v}$ on the product bundle $\mathfrak{M} \times{ }_{M} \mathfrak{C}$ as follows:

$$
\left(L_{P} \mathbf{v}\right)\left(g_{x}, j_{x}^{1} \nabla\right)=s^{g, \nabla}(x)\left(\mathbf{v}_{g}\right)_{x},
$$


and varying $g$ and $\nabla$ independently. The Palatini method can also be applied to other different settings; e.g., see [6], [3], but below we confine ourselves to consider the classical setting for the Palatini method. As is known, the EulerLagrange equations of $L_{P}$ are the vanishing of the Ricci tensor of $g$ (Einstein's in the vacuum) and the condition $\nabla=\nabla^{g}$ expressing that $\nabla$ is the Levi-Civita connection of the metric.

In our case, we can similarly define a first-order Lagrangian $\mathfrak{M} \times_{M} \mathfrak{C}$ by setting $L\left(j^{1} g, j^{1} \nabla\right)=L_{H E}\left(j^{2} g\right)+c\left(\left(\operatorname{alt}_{23}\left(\nabla^{g} T^{g, \nabla}\right)^{\sharp}\right)(\rho \circ g)\right.$. Assuming $M$ is compact, then the action associated with $L$ is given as follows: $\mathcal{S}(g, \nabla)=\epsilon$ $t_{M} L^{\nabla}\left(j^{1} g, j^{1} \nabla\right) \mathbf{v}$, and by considering 1) an arbitrary 1-parameter variation $g_{t}$ of $g$ and 2) the 1-parameter variation $\nabla_{t}=\nabla+t A$ attached to $A \in \Gamma\left(S^{2} T^{*} M \otimes\right.$ $T M)$ of $\nabla$, we obtain, 1) Einstein's equation and 2) $0=\epsilon t_{M} c\left(\operatorname{alt}_{23}\left(\nabla^{g} A\right)^{\sharp}\right) \mathbf{v}_{g}$, $\forall A \in \Gamma\left(S^{2} T^{*} M \otimes T M\right)$, which leads us to a contradiction.

\section{References}

[1] M. Carmeli, Classical fields: general relativity and gauge theory, A WileyInterscience Publication, John Wiley \& Sons, Inc., New York, 1982.

[2] M. Castrillón López, J. Marsden, Some remarks on Lagrangian and Poisson reduction for field theories, J. Geom. Phys. 48 (2003), no. 1, 52-83.

[3] N. Dadhich, J. M. Pons, On the equivalence of the Einstein-Hilbert and the Einstein-Palatini formulations of general relativity for an arbitrary connection, Gen. Relativity Gravitation 44 (2012), no. 9, 2337-2352.

[4] D. B. A. Epstein, Natural tensors on Riemannian manifolds, J. Differential Geometry 10 (1975), 631-645.

[5] P. Von der Heyde, A generalized Lovelock theorem for the gravitational field with torsion, Phys. Lett. A (3) 51 (1975), 381-382.

[6] J. Katz, G. I. Livshits, Affine gravity, Palatini formalism and charges, Gen. Relativity Gravitation 43 (2011), no. 12, 3313-3336.

[7] S. Kobayashi, K. Nomizu, Foundations of differential Geometry, Volume I, John Wiley \& Sons, Inc., N.Y., 1963.

[8] D. Lovelock, The Einstein Tensor and Its Generalizations, J. Mathematical Phys. 12 (1971), 498-501.

[9] L. Mangiarotti, G. Sardanashvily, Connections in classical and quantum field theory, World Scientific Publishing Co., Inc., River Edge, NJ, 2000.

[10] J. Marsden, S. Shkoller, Multisymplectic geometry, covariant Hamiltonians, and water waves, Math. Proc. Cambridge Phil. Soc. 125 (1999), 553-575. 
[11] J. Muñoz Masqué, M. Eugenia Rosado María, Diffeomorphism-invariant covariant Hamiltonians of a pseudo-Riemannian metric and a linear connection, Adv. Theor. Math. Phys. 16 (2012), no. 3, 851-886.

[12] J. Muñoz Masqué, A. Valdés Morales, The number of functionally independent invariants of a pseudo-Riemannian metric, J. Phys. A: Math. Gen. 27 (1994), 7843-7855.

[13] G. A. Sardanashvily, Gauge Theory in Jet Manifolds, Hadronic Press Monographs in Applied Mathematics, Hadronic Press, Inc., Palm Harbor, FL, U.S.A., 1993.

[14] H. Weyl, Space-Time-Matter, translated by H. L. Brose, Dover Publications, Inc., 1952. 